\section{Johannes-Wenner- Forschungspreis}

Der Johannes-Wenner-Forschungspreis hat zum Ziel, die Forschungs- und Projektarbeiten von Mitgliedern der Gesellschaft für Pädiatrische Pneumologie zu unterstützen. Dadurch soll die Versorgung und Behandlung von Kindern mit Lungenerkrankungen verbessert werden. Der Preis wird gemeinsam von der Deutschen Lungenstiftung e.V. und der Gesellschaft für Pädiatrische Pneumologie gestiftet und ausgeschrieben und ist mit $15000 €$ dotiert. Er wird an eine Person oder ein Team vergeben. Das Preisgeld soll der Bearbeitung des beantragten Forschungsprojektes dienen. Der Forschungspreis richtet sich bevorzugt an junge Ärzte und Wissenschaftler, ist aber auch für erfahrene Antragsteller offen. Die Preisverleihung findet auf der Jahrestagung der Gesellschaft für Pädiatrische Pneumologie im März 2016 in Dresden statt. Die Bewerbungsfrist endet am 31. Dezember 2015.

Die Bewerbung muss von einem hauptverantwortlichen Antragsteller eingereicht werden. Lebenslauf und Publikationsverzeichnis der Antragsteller müssen beigefügt werden. Das Forschungsprojekt, für das die Förderung vorgesehen ist, soll in einer 4-seitigen Projektskizze zusammengefasst werden. Diese muss folgende Punkte beinhalten:

- Zusammenfassung des Forschungsvorhabens (max. eine halbe Seite).

- Stand der Forschung und eigene

Vorarbeiten (max. 1 Seite).

- Ziele und Arbeitsprogramm (max. 1,5 Seiten).

- Beantragte Mittel mit Verwendungsnachweis (max. eine halbe Seite).

- Voraussetzungen für die Durchführung des Vorhabens (max. eine halbe Seite).

Die Bewerbung ist entweder als PDF-Datei zu senden an Hansen.Office@mh-hannover.de oder in schriftlicher Form auf dem Postweg einzureichen bei Prof. Dr. med. Gesine Hansen, Medizinische Hochschule Hannover, Klinik für Pädiatrische Pneumologie, Allergologie und Neonatologie, Carl-Neuberg-Straße 1 in 30625 Hannover.

Nach einer Mitteilung der Deutschen Lungenstiftung e.V., Langenhagen

\title{
Bronchiektasien sind keine seltene Erkrankung
}

\author{
Erstmalig liegen nun epidemiologische Daten zur Prävalenz \\ der an Bronchiektasien Erkrankten in Deutschland vor. Sie \\ zeigen, dass es sich nicht, wie vermutet, um ein seltenes \\ Krankheitsbild handelt. Besonders die gehäufte Koinzidenz \\ mit der COPD ist dabei relevant. Die geringen stationären \\ Behandlungsraten legen strukturelle Defizite bei der \\ Betreuung dieser Patienten nahe. \\ Eur Respir J 2015; DOI: 10.1183/13993003.00954-2015
}

Bronchiektasien gelten als relativ selten. Dabei besteht bei diesen Patienten eine sehr hohe individuelle Krankheitslast. Deshalb sind sie auf eine spezifische medizinische Betreuung angewiesen. Um zu prüfen, ob diese in ausreichendem Maß vorgehalten wird, sind epidemiologische Basisdaten (z.B. die Prävalenz) essenziell. Diese lagen in Deutschland bisher nicht vor.

Um eine ausreichend valide Stichprobe zu erhalten, nutzte das Autorenteam um F. C. Ringshausen die Fallzahlen des Risikostrukturausgleichs der öffentlichen Krankenkassen. Bronchiektasien gehören zu den Erkrankungen, in denen dieser Strukturfond greift. Patienten mit Zystischer Fibrose (CF) wurden nicht berücksichtigt, da die Autoren dies als eigenständige Entität werten. Die Hochrechnung der Daten auf die Bevölkerung erfolgte mit den aktuellen demografischen Parametern des Statistischen Bundesamts.

\section{Mehr ältere Patienten betroffen} $\nabla$

In der Datenbank konnten 2595 Patienten mit Bronchiektasie identifiziert werden. Das Durchschnittsalter lag bei 65 Jahren. Die Geschlechterverteilung war balanciert. Hieraus lässt sich hochrechnen, dass in Deutschland rund 53807 Patienten an dieser Krankheit leiden. Dies ergibt eine durchschnittliche Prävalenzrate von 67/100000 Einwohner. Damit erfüllt dieses Krankheitsbild nicht mehr, wie von einigen Autoren vermutet, die Kriterien einer seltenen Erkrankung (Cut off 50/100000). Außerdem fiel auf, dass 58\% der Bronchiektasie-Diagnosen mit einer COPD assoziiert waren. Die höchste Inzi- denzrate fanden die Autoren im Alterssegment 75-84 Jahre, mit 228/100000. Mit zunehmendem Alter nimmt die Inzidenz wieder ab. Die überwiegende Zahl der Patienten wird ambulant betreut. Dies werten die Autoren als Zeichen dafür, dass es in Deutschland an auf dieses Krankheitsbild spezialisierten stationären Betreuungsangeboten mangelt.

\section{Fazit}

Die tatsächliche Prävalenz von Bronchiektasien in der Bevölkerung wurde bisher unterschätzt, so die Autoren der vorliegenden Studie. Erstmalig liegen nun epidemiologische Daten vor, die zeigen, dass ein relativ großer Teil der Bevölkerung betroffen ist und eine Assoziation mit der COPD besteht. Bei Patienten im Alter zwischen 75 und 84 Jahren muss besonders auf diese Problematik geachtet werden. Die relativ geringen stationären Fallzahlen indizieren einen Handlungsbedarf bei der Optimierung der Versorgungsstruktur.

\section{Dr. Horst Gross, Berlin}

\section{Erratum}

Auf der Titelseite der August-Ausgabe 2015 der Pneumologie hat sich der Fehlerteufel eingeschlichen. Richtigerweise heißt es „Pleuraempyem in der Postpneumonektomiehöhle“. Wir bitten dies zu entschuldigen. 